\title{
Rapid Deployment of Curved Surfaces via Programmable Auxetics
}

\author{
MINA KONAKOVIĆ-LUKOVIĆ, EPFL \\ JULIAN PANETTA, EPFL \\ KEENAN CRANE, Carnegie Mellon University \\ MARK PAULY, EPFL
}

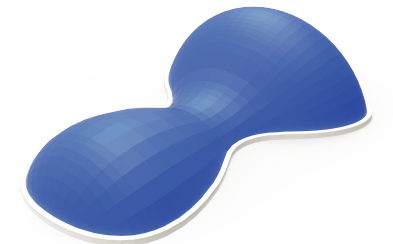

input design

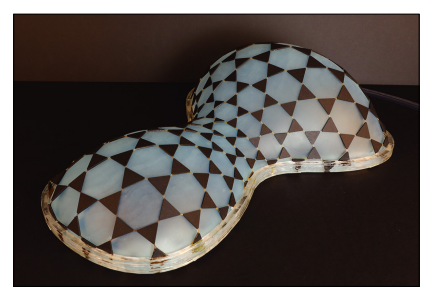

deployed structure

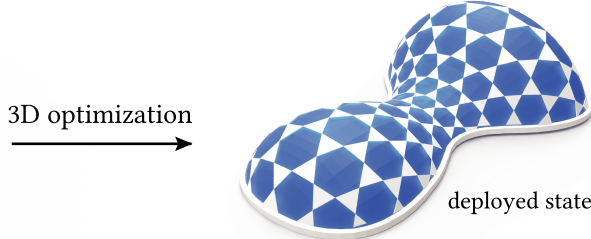

optimized auxetic linkage

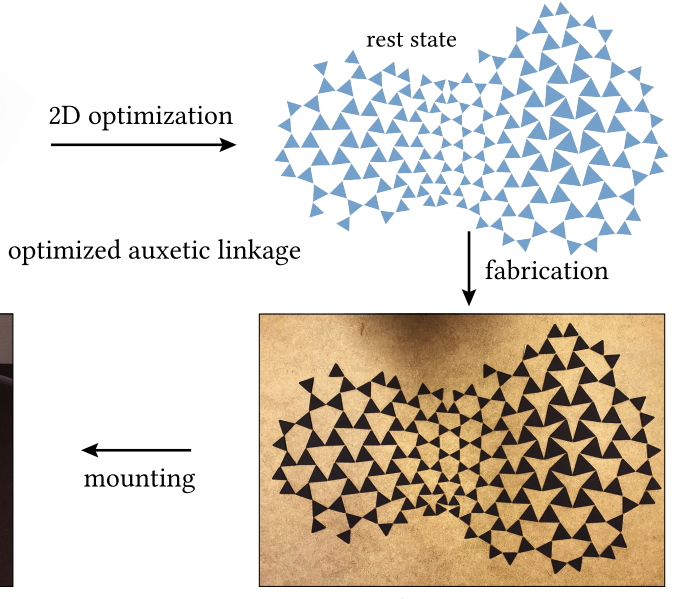

flat material

Fig. 1. Our algorithm computes a triangular auxetic linkage that closely approximates a given surface when deployed to maximal extension via inflation. The fabricated material is laser cut from a single sheet, mounted onto the support frame, and inflated with a generic rubber balloon.

Deployable structures are physical mechanisms that can easily transition between two or more geometric configurations; such structures enable industrial, scientific, and consumer applications at a wide variety of scales. This paper develops novel deployable structures that can approximate a large class of doubly-curved surfaces and are easily actuated from a flat initial state via inflation or gravitational loading. The structures are based on two-dimensional rigid mechanical linkages that implicitly encode the curvature of the target shape via a user-programmable pattern that permits locally isotropic scaling under load. We explicitly characterize the shapes that can be realized by such structures-in particular, we show that they can approximate target surfaces of positive mean curvature and bounded scale distortion relative to a given reference domain. Based on this observation, we develop efficient computational design algorithms for approximating a given input geometry. The resulting designs can be rapidly manufactured via digital fabrication technologies such as laser cutting, CNC milling, or 3D printing. We validate our approach through a series of physical prototypes and present several application case studies, ranging from surgical implants to large-scale deployable architecture.

Authors' addresses: Mina Konaković-Luković, mina.konakovic@epfl.ch, EPFL, Route Cantonale, Lausanne, Switzerland, 1015; Julian Panetta, EPFL, julian.panetta@epfl.ch; Keenan Crane, Carnegie Mellon University, 5000 Forbes Avenue, Pittsburgh, Pennsylvania, 15213, kmcrane@cs.cmu.edu; Mark Pauly, EPFL, mark.pauly@epfl.ch.

Permission to make digital or hard copies of all or part of this work for personal or classroom use is granted without fee provided that copies are not made or distributed for profit or commercial advantage and that copies bear this notice and the full citation on the first page. Copyrights for components of this work owned by others than ACM must be honored. Abstracting with credit is permitted. To copy otherwise, or republish, to post on servers or to redistribute to lists, requires prior specific permission and/or a fee. Request permissions from permissions@acm.org.

(C) 2018 Association for Computing Machinery.

0730-0301/2018/8-ART106 \$15.00

https://doi.org/10.1145/3197517.3201373
CCS Concepts: • Computing methodologies $\rightarrow$ Mesh geometry models; • Applied computing $\rightarrow$ Computer-aided design; Computer-aided manufacturing;

Additional Key Words and Phrases: computational fabrication, smart materials, digital fabrication, auxetic materials, conformal geometry

\section{ACM Reference Format:}

Mina Konaković-Luković, Julian Panetta, Keenan Crane, and Mark Pauly. 2018. Rapid Deployment of Curved Surfaces via Programmable Auxetics. ACM Trans. Graph. 37, 4, Article 106 (August 2018), 13 pages. https://doi.org/ 10.1145/3197517.3201373

\section{INTRODUCTION}

Deployable structures are shape-shifting mechanisms that can transition between two or more geometric configurations. Often conceived to minimize space requirements for storage or transport, deployable structures are used, for example, for antennas or solar panels in satellites, as coronary stents in medical applications, as consumer products (e.g. umbrellas), or in architectural designs (e.g. retractable bridges or relocatable, temporary event spaces).

Most existing realizations of deployable structures are geometrically simple and often exhibit strong symmetries. Deploying more general curved surfaces is made difficult by the inherent complexity of jointly designing initial and target geometries within the constraints imposed by the deployment mechanism [Gantes 2001].

We propose a new class of deployable structures and associated computational methods that enable rapid deployment of doublycurved freeform surfaces (see Figure 1). Our approach is based on a 
planar linkage of rigid triangles connected by rotational joints at vertices; this linkage has regular connectivity, but spatially varying scale. In-plane rotation of the triangles induces an approximately isotropic expansion or contraction in area, which allows a mechanical interpretation of the linkage as an auxetic surface metamaterial [Saxena et al. 2016], or a geometric interpretation in terms of conformal maps [Konaković et al. 2016]. By spatially varying the triangle sizes, we effectively control the maximum possible expansion at each point, which in turn provides control over curvature: under maximal extension, nonuniform expansion forces the structure to buckle out of the plane and assume a curved configuration. Here several questions arise: which curvature functions can be encoded in such a pattern? How can we actuate a linkage to achieve maximal expansion? Which surfaces can we hope to realize using this procedure? Several key contributions help to address these questions:

- We introduce spatially graded auxetic metamaterials suitable for deployment via inflation or gravitational loading. In particular, we show that these deployment strategies achieve maximal expansion everywhere and provide additional regularization to ensure that the target shape is unique.

- We provide a general analysis of deformation by inflation and gravitational loading to formally classify the set of realizable doubly-curved target shapes.

- We present an optimization algorithm to solve the inverse design problem: Given a desired target geometry, our method finds appropriate scaling parameters and a corresponding layout of the 2D linkage such that the target shape is achieved when the linkage is deployed.

The resulting structures offer a number of benefits: (i) The reststate is (piece-wise) flat, which facilitates compact storage as well as cost- and time-efficient fabrication techniques (such as laser cutting or milling); (ii) The target geometry is directly encoded in the 2D linkage structure so that no additional support or scaffolding is required to guide deployment; (iii) Our approach is scale-invariant and can be applied to realize a broad and explicitly defined class of doubly-curved surfaces. If a given surface is not within the set of realizable shapes, we apply optimization to find a feasible target surface that is close to the desired design.

The rest of the paper is organized as follows: Section 2 reviews connections to related work. Section 3 considers geometric models of inflation and gravitational loading, helping to understand the feasible design space. Specifically, we characterize the shapes that can be achieved via inflation or gravitational loading in terms of surfaces of positive mean curvature and conformal deformations with bounded scale factor. Section 4 introduces our spatially graded auxetic metamaterial, realized as a rigid triangular linkage. We show how to locally adapt maximal expansion (and hence, target curvature) by varying the scale and orientation of linkage elements in the initial flat state. Section 5 describes an optimization algorithm for solving the inverse design problem, i.e., finding suitable parameters for our metamaterial that ensure the target surface is faithfully approximated when actuated. In Section 6 we present several case studies and physical prototypes that highlight potential applications across domains ranging from small-scale heart stents to large-scale
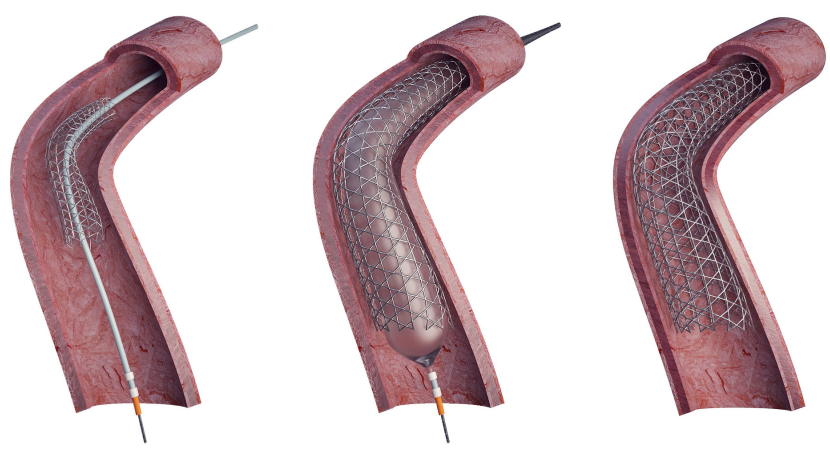

Fig. 2. Conventional heart stents are straight and typically chosen by the surgeon from a set of standard sizes. Recent research has shown the benefits of curved stents [Tomita et al. 2016]. Our method can be used to create freeform curved heart stents that can be adapted to the specific geometry of the patients' blood vessels. The stent is administered with a catheter to the correct position (left) inflated to its target geometry (middle, right).

air-supported domes. We conclude with a discussion of the limitations of our approach, and also identify opportunities for future research (7).

\section{RELATED WORK}

Computational material design. Several previous works have designed custom materials to achieve high-level deformation goals. Bickel et al. [2010] stack layers of various nonlinear base materials to produce a desired force-displacement curve. Microstructure design works [Panetta et al. 2015; Schumacher et al. 2015; Zhu et al. 2017] construct small-scale structures from one or two printing materials to emulate a large space of linearly elastic materials. These works focus on designing deformable materials that typically undergo small stretches and return to their rest configurations when unloaded, making them less suitable as deployment mechanisms.

Inverse elastic shape design. Another common goal is to optimize deformable objects' rest shapes so that they assume desired equilibrium shapes under load. The inverse elastic shape design algorithms of [Chen et al. 2014; Pérez et al. 2015] design flexible objects achieving specified poses under gravity or user-defined forces. These works do not attempt to find compact rest configurations amenable to efficient fabrication, transport, and deployment. One exception is [Skouras et al. 2012], which designs rubber balloons that inflate to desired target shapes. However, fabricating custom rubber balloons involves a complicated multi-step molding process best suited for small-scale target shapes. Additionally, the inflation must be carefully controlled to avoid under- or over-inflating. In contrast, our flat initial state facilitates simple fabrication at a wide range of scales. Our deployment method is also more robust, since the final state is precisely singled out by construction - the target is reached when the material cannot expand any further.

Deployment-aware design. Other works have focused on designing objects that rapidly expand into nearly rigid target shapes. Skouras et al. [2014] construct inflatable structures by fusing together sheets of nearly inextensible material. Because each panel inflates 


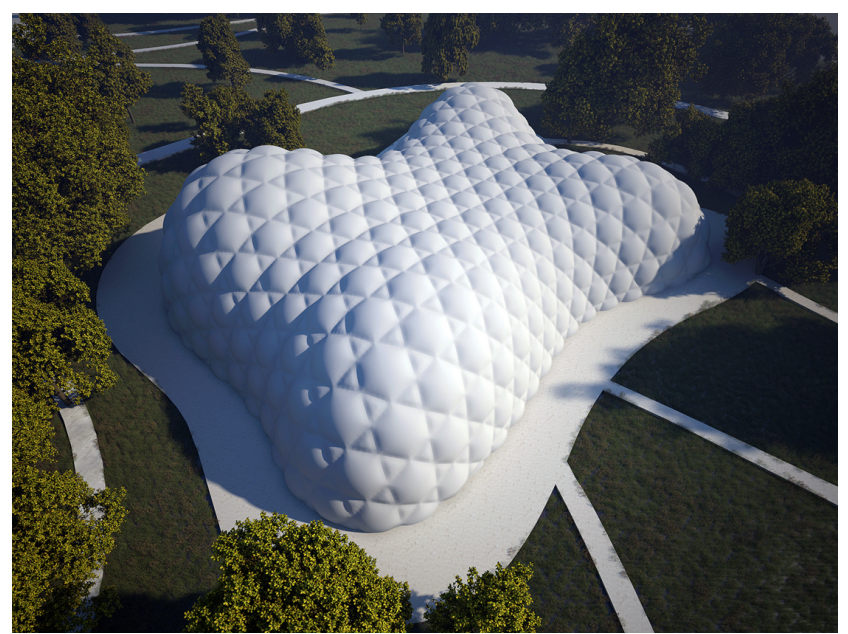

Fig. 3. Design study of deployable architecture. The freeform inflatable dome can be used as a semi-permanent, relocatable space.

into a nearly developable surface, many small panels are potentially needed to closely approximate a smooth, wrinkle-free doublycurved surface. Zheng et al. [2016] design compact scissor linkage assemblies that, when stretched, uniformly expand into coarse approximations of 3D shapes. Their method ensures a collision-free expansion path for sparse wireframe designs. Dudte et al. [2016] perform basic research into approximating singly- and doubly-curved surfaces with generalized Miura folds. Their origami patterns have a single degree of freedom parametrizing their path from the flat configuration to the target shape. For doubly curved surfaces, the construction is bi-stable, leading to an especially simple deployment process. However, the design algorithm produces flat configurations with over twice the surface area of the target.

Actuated shape-shifting. The engineering and graphics communities have both sought to design mechanisms that transition between discrete configurations or trace out continuous deformation paths when actuated. By embedding a rigid fiber lattice in flexible silicon, Connolly et al. [2017] design tubes that accurately reproduce bending and twisting motions when inflated. Ma et al. [2017] generalize this idea, segmenting objects into chambers that, when inflated to certain pressures, drive the shape into a sequence of desired poses. Also using pneumatic actuation, Overvelde et al. [2016] present an origami-inspired metamaterial that dramatically changes shape, and Ou et al. [2016] design flat sheets that fold into complex origami shapes. Raviv et al. [2014] design structures that can bend, stretch, and fold when exposed to water. Liu et al. [2017] study how a pre-strained elastomer sheet patterned with ink can self-fold when heated by a lamp.

Actuated form-prescribed geometry. Recent work [Guseinov et al. 2017; Pérez et al. 2017] follows a similar rationale of encoding a $3 \mathrm{D}$ target surface in a flat sheet of material. In these methods, the activation mechanism is directly integrated into the material in the form of a pre-tensioned elastic membrane. Upon release, the membrane contracts and forces the pre-shaped rigid elements into their global target configuration. This approach achieves impressive results, but has several drawbacks. (i) Pre-stretched materials are limited in scale. (ii) Fabrication is more complex, since it requires compositing multiple materials. (iii) Shaping by contraction means that the flat surface is larger in area than the target surface, reducing potential packing benefits. (iv) Closed surfaces are more difficult to realize (only disk-topology surfaces have been shown).

Our approach is scale-invariant, does not require multi-material compositing (our inflation balloons need not be attached to the auxetic linkage), leads to compact flat-packed sheets, and can handle shapes of arbitrary topology.

Auxetic Materials. Auxetic surface materials are an essential ingredient of our approach: auxetic linkages permit otherwise inextensible flat sheets of material to uniformly stretch as needed to deform into doubly curved surfaces. We refer the reader to [Saxena et al. 2016] for a survey on auxetic patterns, their unique mechanical properties, and their potential applications to diverse engineering and medical problems. In graphics, Konaković et al. [2016] introduced a design tool for fabricating curved target surfaces by cutting auxetic patterns into flat sheets. However, the resulting uniform linkage pattern is difficult to deploy because the target surface is not singled out in any way; the structure can just as easily deform into an infinite family of other surfaces. This ambiguity necessitates the use of guide surfaces and careful manual alignment when shaping the material. Our work addresses this limitation by spatially varying the pattern to uniquely encode the target shape, enabling rapid deployment without guide surfaces by simple expansion.

Friedrich et al. [2018] also seek to encode the target surface by limiting the pointwise maximal stretch factors. Rather than designing a fully opened linkage on the target surface, the authors outline a heuristic to construct a partially opened pattern in the plane: based on the scale factors of a conformal map, they insert polygonal openings resembling those at the top of Figure 6. However, it is unclear how these polygonal openings are positioned and connected to ensure proper linkage functionality. The authors then propose an iterative evolutionary optimization process needed to bring the fully opened pattern closer to the target surface. In contrast, our algorithm directly ensures the target surface is closely approximated by the fully opened linkage; furthermore, we detail efficient mechanisms for deployment and characterize the space of achievable designs for each deployment mechanism.

Multi-stable auxetic patterns [Rafsanjani and Pasini 2016] are another potential avenue for encoding maximal stretch factors in a material: they expand from their rest configuration and settle into stable equilibrium at one or more stretched configurations. We leave investigation on how to modify these patterns to encode curved surfaces as future work.

Polyhedral Patterns. When deployed, our auxetic linkage's equilateral triangles and hexagonal openings tile the target surface with a tri-hex pattern. A common task in architectural geometry is to rationalize curved input surfaces using planar polyhedral patterns. Schiftner et al. [2009] and Jiang et al. [2015] both introduce algorithms that can approximate input surfaces with tri-hex patterns as a special case. Vaxman et al. [2017] propose a form-finding tool for general combinatorial patterns and show applications to tri-hex 
meshes. These works focus on symmetry, planarity, and other pattern quality requirements, making no attempt to ensure the tri-hex pattern can be flattened into a planar configuration by closing the hexagons, which is essential in our approach.

\section{SHAPE SPACE}

Which shapes can we hope to achieve with our structures? The answer depends jointly on the geometry of the structure, as well as the method used to actuate it. Rather than study this question in terms of the detailed geometry of a specific mechanical linkage, we will first consider an idealized model based on smooth differential geometry. This analysis will then inform the design of discrete mechanical linkages and their physical actuation described in Section 4 and the corresponding optimization algorithm discussed in Section 5. In particular, we will explicitly characterize the shapes one can hope to achieve via (i) inflation and (ii) gravitational loading; we will also make an interesting connection between inflated balloons and conformal geometry (Section 3.2.1).

\subsection{Preliminaries}

In this section, we consider a closed, compact, and oriented topological surface $M$ with geometry given by a map $f: M \rightarrow \mathbb{R}^{3}$ assigning coordinates to each point of $M$. The differential $d f$ of $f$ maps tangent vectors $X$ on $M$ to the corresponding vectors $d f(X)$ in $\mathbb{R}^{3}$; the differential is also sometimes denoted as the facobian or deformation gradient. A map $f$ is an immersion if its differential is injective, i.e., if at each point $p \in M$ it maps nonzero vectors to nonzero vectors; since $M$ is compact, it is an embedding if $f$ is also injective (loosely speaking: if it has no self-intersections). Formally, we will require that $f$ is a twice differentiable immersion with bounded curvature.

To any immersed surface we can associate the quantity

$$
\operatorname{vol}(f):=\int_{M} N \cdot f d A_{f},
$$

where $N$ is the outward unit normal, and $d A_{f}$ is the area element induced by $f$; when $f$ is embedded, $\operatorname{vol}(f)$ is just the enclosed volume. We will also use $g$ and $H$ to denote the metric and mean curvature (resp.) induced by $f$. We use the definition $H=\frac{1}{2} \nabla_{f} \cdot N$, so, e.g., a sphere has constant positive mean curvature. If $d A$ and $d \tilde{A}$ are two area measures on $M$, we will write $d A \leq d \tilde{A}$ to mean that $d A(U) \leq d \tilde{A}(U)$ for all open sets $U \subset M$. When considering variations of the surface, we will think of $f$ as a time-parameterized family of immersions $f(t)$ and adopt the shorthand $\dot{\phi}:=\left.\frac{d}{d t} \phi\right|_{t=0}$ for any time-varying quantity $\phi$.

\subsection{Inflation}

To understand the space of shapes that can be achieved via inflation, we consider an idealized and purely geometric model of rubber balloons. From a mechanical viewpoint, our model would correspond (very roughly) to a thin isotropic elastic membrane with spatially varying maximal expansion. This model should however be taken with a grain of salt: our goal here is not to formulate a precise mechanical model, but rather to get a sense of the most significant geometric effects exhibited by our discrete mechanism-a more rigorous analysis (e.g., based on homogenization of the small-scale

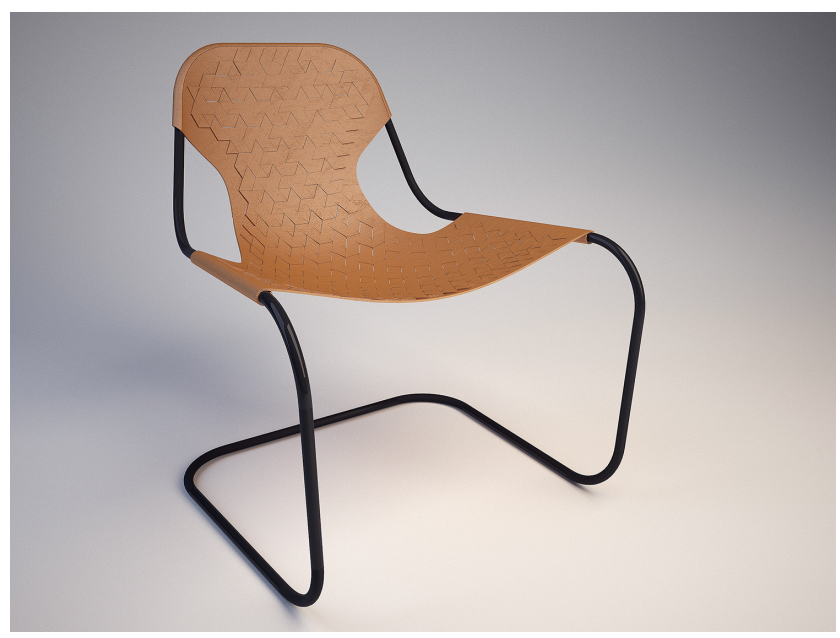

Fig. 4. Design study of a freeform chair realized using four layers of spatially graded auxetic material to fully cover the surface (see Section 5 for details).

geometry) is beyond the scope of this paper. Moreover, for computational design, it is often more useful to have a simple and easily computable geometric model than a detailed mechanical model which is accurate but difficult to explore due to heavy computational requirements (e.g., finite element analysis).

We specifically consider the geometry of immersions that (i) locally maximize enclosed volume, and (ii) do not stretch area above a given upper bound. Note that we do not consider questions of $d y$ namics (e.g., "can this configuration be reached from a given starting point?"), which are notoriously difficult even without constraints on volume or area. Instead we consider only the simple static question of, "what will be true about a surface that achieves these conditions?" In particular, we make the following observation:

Proposition 3.1. Let $d A^{+}$be an area measure on M. Among all (twice differentiable) immersions $f: M \rightarrow \mathbb{R}^{3}$ such that $d A_{f} \leq d A^{+}$, those that locally maximize the enclosed volume vol $(f)$ will (i) have strictly positive mean curvature $H>0$ away from sets of measure zero, where $H \geq 0$; and (ii) will achieve the upper bound on area $\left(d A_{f}=d A^{+}\right)$.

Proof. (i) Suppose an immersion $f$ admits a nonempty open set $D \subseteq M$ on which $H \leq 0$. Then we can construct a smooth positive function $u: M \rightarrow \mathbb{R}$ supported on $D$ and consider the outward normal variation $\dot{f}:=u N$. The corresponding first-order changes in volume and area measure are given by

$$
\begin{aligned}
\left.\frac{d}{d t} \operatorname{vol}(f)\right|_{t=0} & =\int_{M} u d A_{f}>0 \quad \text { and } \\
\left.\frac{d}{d t} d A_{f}\right|_{t=0} & =2 u H d A_{f},
\end{aligned}
$$

respectively. Since $u H \leq 0$, this variation increases volume without increasing area; hence, $f$ is not a volume maximizing immersion. Moreover, if $H<0$ at any point $p \in M$, then (by continuity of $H$ ) there must be an open ball around $p$ on which $H$ is strictly negative. Hence, on sets of measure zero, an immersion $f$ that maximizes volume must have $H \geq 0$. 


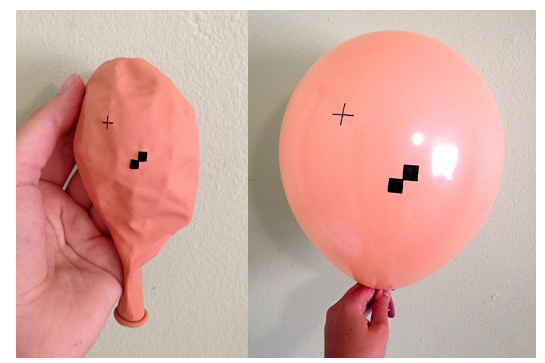

Fig. 5. When inflated, rubber balloons exhibit near-conformal deformation (indicated by the preservation of right angles), further motivating our use of an auxetic design space for inflatable structures.

(ii) Since both $d A_{f}$ and $d A^{+}$are area measures, we have $d A^{+}=$ $\varphi d A_{f}$ for some continuous function $\varphi: M \rightarrow \mathbb{R}$. If $d A_{f}<d A^{+}$, then there will be at least one point $p \in M$ where $\varphi(p)<1$, and by continuity, an open neighborhood $D$ around $p$ where $\varphi<1$. Letting $u$ be a smooth positive function supported on $D$, a normal variation $u N$ will now increase the volume without violating the area bound.

Roughly speaking, the surfaces that can be realized via inflation in our model are those that have positive mean curvature (see Proposition 3.1 and Section 4.1 for further discussion). In practice, we therefore modify a given target surface to have positive mean curvature, as described in Section 3.4.

\subsubsection{Conformal Balloons.}

Suppose we no longer consider volume maximization nor an upper bound on area, but simply ask about the shape of a balloon that tries to minimize the elastic membrane energy when filled with a fixed volume of air. In particular, the Dirichlet energy $E_{D}(f):=$ $\int_{M}|d f|^{2} d A_{0}$ models an elastic membrane with zero rest length, or, asymptotically, the energy due to extreme stretching. Critical points of Dirichlet energy are called harmonic maps, and any harmonic map between topological spheres is necessarily holomorphic or antiholomorphic [Eells and Wood 1976]. We hence find a connection to conformal geometry:

Theorem 3.2. Consider a surface $\left(M, g_{0}\right)$ of spherical topology. Among all embeddings $f: M \rightarrow \mathbb{R}^{3}$ of fixed enclosed volume vol $(f)=$ $c$, any embedding minimizing the membrane energy $E_{D}$ is conformal, i.e., the induced metric $g:=d f \otimes d f$ is conformally equivalent to $g_{0}$.

Proof. Let $\tilde{f}$ be a non-conformal embedding with volume $c$, and let $\Sigma:=\tilde{f}(M) \subset \mathbb{R}^{3}$ denote the image of $\tilde{f}$. We know that a map minimizing Dirichlet energy over all orientation-preserving embeddings $f:\left(M, g_{0}\right) \rightarrow \Sigma$ is holomorphic (and in particular, conformal since it is injective and orientation-preserving). Therefore, because $\tilde{f}$ is not conformal, we can find an embedding $f^{*}$ mapping to $\Sigma$ with smaller Dirichlet energy. This $f^{*}$ still has volume $c$, but lower $E_{D}$, so $\tilde{f}$ is not minimal.

Due to the rather simplistic model of membrane energy, one might wonder whether this theorem provides any useful information about real physical balloons or our auxetic mechanisms (which allow an area scaling of at most 4). However, Dirichlet energy will still tend to dominate more realistic nonlinear models of elasticity in the limit of large stretching. Consider for instance the elastic energy described by Chao et al. [2010], $E(f):=\int_{M}\left|d f-R_{f}\right|^{2} d A$, where $R_{f}$ denotes the rotation closest to $d f$. In the limit of large strain, the quadratic (i.e., Dirichlet) term of the expansion $|d f|^{2}-2\left\langle d f, R_{f}\right\rangle+$ $\left|R_{f}\right|^{2}$ dominates, and we are left with the same picture as before. Moreover, the nonlinear terms drop off rather quickly, suggesting that one should observe conformal behavior even for moderate stretching-as supported by physical experiments such as the one pictured in Figure 5. This observation further motivates our use of auxetic materials with bounded scale factors for inflatable structures.

\subsection{Gravitational Deployment}

Gravity is an even simpler mechanism for shape deployment: just suspend a sheet of material by its boundary and let gravity pull it into the target shape. This approach is most suitable for surfaces with simple boundary curves like in Figure 11. In fact, to simplify the fabrication process, we require that the initial surface spanning the boundary curves be a height field; otherwise attaching the flat material to the boundary curves would require a complicated manual deformation. The height field property also guarantees that the downward gravitational force has a positive component along the surface's normal direction, ensuring that it can pull the surface open analogously to the inflation setup.

When fabricated from our idealized material (characterized by having zero stiffness until an upper area bound is reached), we observe that height-field-initialized surfaces will remain height fields during the deployment. This follows from the fact that only two types of forces act on interior points during the deployment: gravity and the material stresses enforcing the area bound. Gravity pushes points in the material straight downward, decreasing height values but preserving the height field property. Stresses enforcing the area stretch bound always take the form of tensile forces: regions of material that have reached their stretching bound pull uniformly inward against the surrounding material (tangentially to the surface). Unlike expansive forces, these tensile forces act to straighten out the material and will not cause the sheet to fold over itself to violate the height field property.

We now characterize the space of height field surfaces deployable by gravity, again ignoring questions of dynamics. For consistency with the inflation setup, we orient the surface's height axis vertically (parallel to gravity) and choose the surface orientation so that normals point downward. The gravitational deployment process is formulated as minimizing the immersion $f$ 's gravitational potential energy:

$$
U(f):=\int_{M} f \cdot z d A
$$

where $z$ is the height axis vector oriented opposite gravity and scaled by the gravitational acceleration constant. Note that $d A$ is the area element induced by an isometric immersion of $M$ (for which we assume the material density is 1) and is independent of the particular immersion $f$. 
Proposition 3.3. A height field surface represented as a smooth immersion $f: M \rightarrow \mathbb{R}^{3}$ that locally minimizes the gravitational potential energy $U(f)$ over all smooth immersions satisfying $d A_{f} \leq d A^{+}$ and Dirichlet conditions $f=f_{\text {tgt }}$ on $\partial M$, must (i) have strictly positive mean curvature $H>0$ away from sets of measure zero, where $H \geq 0$; and (ii) achieve the upper bound on area $\left(d A_{f}=d A^{+}\right)$.

Proof. (i) Suppose there exists a region $D \subseteq M$ of nonzero measure on which $H \leq 0$. We can construct a smooth, positive bump function $u$ compactly supported on $D$ so that the positive normal variation $\dot{f}:=u N$ decreases gravitational potential to first order:

$$
\left.\frac{d}{d t} U(f+t u N)\right|_{t=0}=\int_{D} u N \cdot z d A<0,
$$

because $N \cdot z<0$ by the height field property. Furthermore, this variation does not violate the upper bound on area: the area measure changes by

$$
d \dot{A}_{f}=2 u H d A_{f} \leq 0 .
$$

Therefore, $f$ does not locally minimize gravitational potential energy. (The proof of part (ii) is analogous to Proposition 3.1.)

\subsection{Projection to Feasible Surfaces}

If the surface violates the positive mean curvature requirement, we must modify it for compatibility with our deployment mechanisms. However, we wish to keep the design as similar to the input surface as possible. Accordingly, we change the surface only where needed, leaving the regions of positive mean curvature untouched. In the regions violating the requirement, we make the smallest change necessary in mean curvature space.

We propose the following repair process to achieve these goals: apply mean curvature flow $\dot{f}=-H N$ to each region of negative mean curvature, terminating when mean curvature reaches zero. Then, to ensure $H \geq \varepsilon>0$, an arbitrarily small, smooth normal variation can be applied, computed, e.g., by solving Equation 1 with $\dot{H}=1$ and zero Dirichlet boundary conditions.

Our repair process indeed produces the closest admissible surface in the sense of minimizing pointwise curvature distance $\left|H-H_{0}\right|$ almost everywhere in $M$ (where $H_{0}$ is the mean curvature of the initial immersion): it preserves mean curvature in the positive regions and minimally adjusts each non-positive value. Curvature-based distance metrics like this are often considered good models of perceptual distance [Kim et al. 2002]. However, for the examples we tried, we can make an additional observation: the repair process also locally minimizes pointwise distances to the original surface.

We formalize the repair process as follows. For a smooth initial immersion $f_{0}: M \rightarrow \mathbb{R}^{3}$, the regions $R_{i} \subset M$ on which $H<0$ are always bounded by well-defined curves $\partial R_{i}$. The repair process cuts away each $f_{0}\left(R_{i}\right)$ and replaces it with a minimal surface $f\left(R_{i}\right)$ spanning the same immersed boundary curve. This viewpoint corresponds to the limit $\varepsilon \rightarrow 0$.

First, we consider the space of admissible variations one might apply to the repaired surface when attempting to move it closer to the original. We consider an arbitrary suitably regular variation $\dot{f}:=R_{i} \rightarrow \mathbb{R}^{3}$ and define normal velocity $u:=\dot{f} \cdot N$ for convenience.
We observe that $u=0$ on $\partial R_{i}$ since the perturbed surface must still fill the same boundary curve.

The corresponding first-order change in mean curvature is [Doğan and Nochetto 2012]:

$$
2 \dot{H}=-\Delta_{f} u-\left(\kappa_{1}^{2}+\kappa_{2}^{2}\right) u+2 \dot{f} \cdot \nabla_{f} H=-\Delta_{f} u-2|K| u,
$$

where $\kappa_{1}=-\kappa_{2}$ are the minimal surface patch's principal curvatures. The term involving $\dot{f}$ vanishes because $H \equiv 0$, and we applied the simplification $\kappa_{1}^{2}+\kappa_{2}^{2}=2\left|\kappa_{1} \kappa_{2}\right|=2|K|$. Preserving non-negative mean curvature requires:

$$
\dot{H} \geq 0 \quad \Longrightarrow \quad \triangle_{f} u+2|K| u \leq 0 .
$$

For small $|K|$ (mildly curved repaired patches), we expect the Laplacian term to dominate and force the normal velocity to achieve its minimum on the boundary $\partial R_{i}$ (superharmonic functions obey a minimum principle). But $u=0$ there, forcing $u \geq 0$ inside $R_{i}$.

Furthermore, in our experiments, closest points on the original surface always lie to the negative side of the repaired patch in that, $\forall p \in f\left(R_{i}\right)$ and nearest original points $p^{*}=\operatorname{argmin}_{\tilde{p} \in f_{0}\left(R_{i}\right)}\|\tilde{p}-p\|$, we have $N \cdot\left(p^{*}-p\right) \leq 0$. This should be expected for moderate edits, as the curvature flow process converging to the minimal surface moves points only in the positive normal direction. In these cases, moving any point on our repaired surface closer to the original surface requires a motion in the negative normal direction which, for small $|K|$, violates the non-negative mean curvature constraint.

\section{MATERIAL DESIGN}

Our goal is to design a mechanism that deforms from an initial flat configuration into a doubly-curved target surface when actuated by inflation or gravity. Konaković et al. [2016] study a similar problem, where linkages based on the regular 2D Kagome lattice are deformed into general curved target shapes. This approach has two key obstacles to overcome when it comes to rapidly deployable structures, namely (i) a perfectly regular lattice encodes no information about the target shape, necessitating some kind of "scaffolding" such as a 3D print to guide assembly, and (ii) there is no clear way to actuate such a surface, which must be laboriously pointwise-aligned to the mold and deformed by hand. These observations motivate us to (i) encode the target shape into the linkage by considering a spatially varying pattern rather than a regular one, and (ii) consider geometries that can be rapidly deployed via inflation or gravity, as studied in Section 3.

Discrete Conformal Geometry. A key motivation for starting with the Kagome lattice is that, as observed by Konaković et al. [2016], deformations of this lattice behave at the large scale like conformal mappings with bounded scale factor. This loose analogy is made a bit more precise by making a connection to the Cauchy-Riemann equations: for both conformal maps and the lattice, infinitesimal planar motions are determined by real degrees of freedom at the boundary. Another connection recently made by Lam [2017] is that infinitesimal rotations of the lattice can be described as discrete harmonic functions (in the usual sense of the cotangent Laplacian), mirroring the fact that for the logarithmic derivative $\log \left(z^{\prime}\right)=u+\imath \theta$ of a holomorphic map $z$, the two components $u, \theta$ describing scaling and rotation (resp.) are conjugate harmonic. To date, however, there 


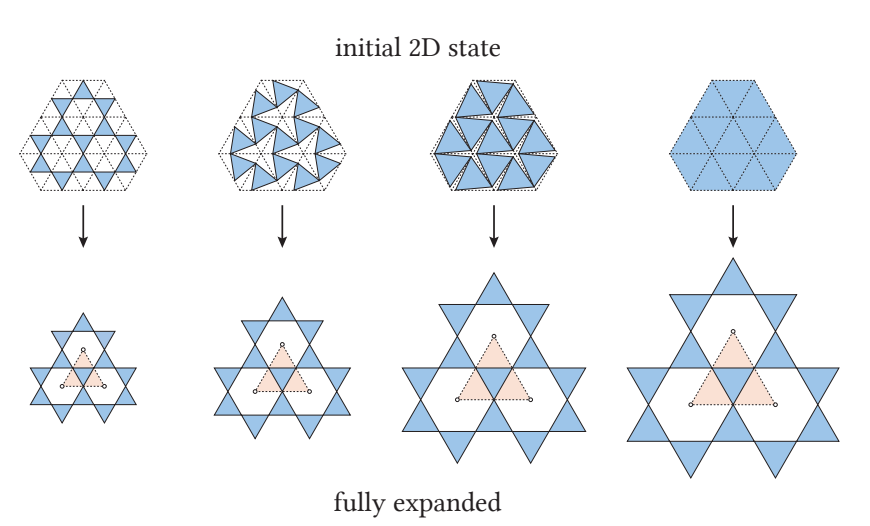

Fig. 6. Spatially variable maximal expansion of the linkage can be achieved by scaling and rotating the linkage triangles in the initial $2 \mathrm{D}$ state. When already fully opened (left), no more expansion is possible. When fully closed (right), the linkage can expand to increase by a factor two in length (or a factor of four in area). Partially opening the initial configuration allows varying the scale factor, indicated by the size of the orange triangles connecting the barycenters of the openings.

is still no complete discrete theory of conformal maps based on the Kagome lattice that includes finite deformations, nor conformal immersions in $\mathbb{R}^{3}$. Nonetheless, adopting the conformal point of view allows us to leverage well-developed tools from computational conformal geometry for the purpose of designing deployable mechanisms.

Mechanical Properties. From a mechanical point of view, linkages based on the Kagome lattice are flexible enough to produce a wide variety of curved surfaces and already have a locking mechanism built-in: stretching the material to four times its original area fully opens the linkage, blocking further expansion. In fact, one can easily show that the linkage is rigid (albeit unstable) in its fully open configuration; additional forces such as gravity or air pressure help to stabilize the fully open state. We take advantage of these mechanical properties to aid deployment. In particular, we adapt the pattern to achieve a spatially varying (rather than constant) maximum bound on expansion across the surface. When deployed, the varying expansion leads to out-of-plane buckling; thus the linkage must assume a curved configuration.

The geometric and mechanical pictures can of course be linked: the bound on expansion in the discrete linkage can be modeled by a bound on the conformal scale factor $e^{u}$ of a smooth conformal map, and the buckling exhibited by the deployed linkage is approximately determined by the Yamabe equation $\Delta u=e^{2 u} K$ relating the logarithm of the scale factor to the Gaussian curvature $K$ of a smooth surface approximating the target geometry. To explore designs for our mechanical linkage, we therefore adopt a strategy based on geometry: first, we compute a conformal map from the plane to the target surface, and read off the scale factors $\lambda_{\text {tgt }}:=e^{u}$. We then use these factors to design or "program" a spatially-graded pattern that approximately matches the corresponding maximum expansion at each point. When fully expanded, a mechanism based on this pattern should approximate the desired target shape. Below
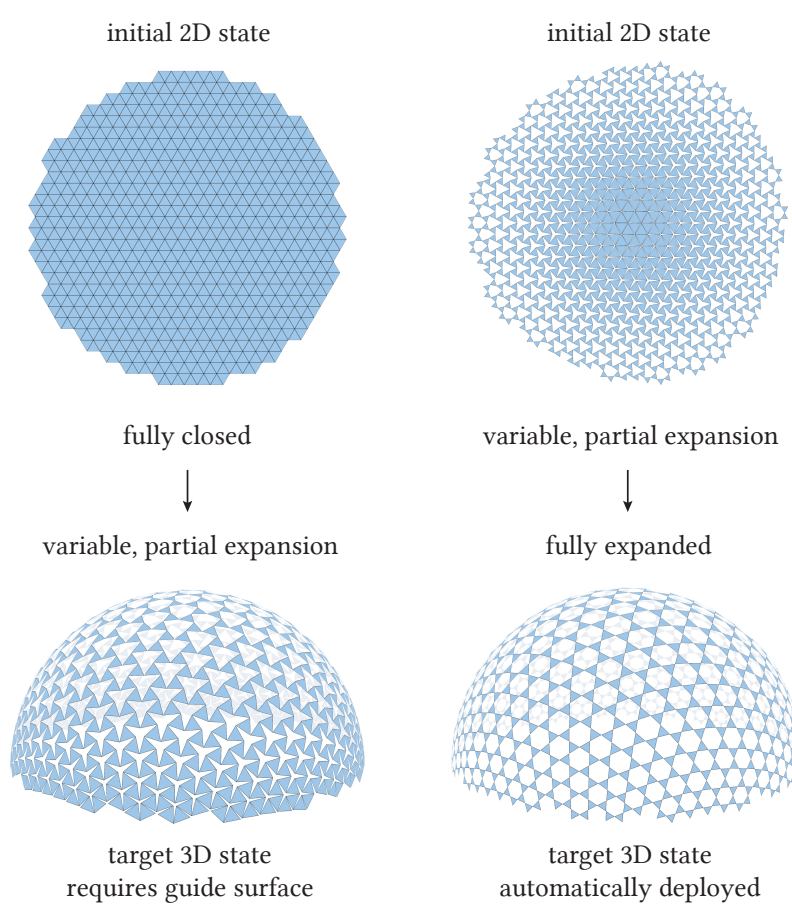

Fig. 7. The method of Konaković et al. [2016] (left) uses a uniform, fully closed initial 2D state and achieves its target state with variable partial openings. Proper deployment thus requires a guide surface and precise manual alignment. In contrast, our spatially varying initial openings in the 2D state allow encoding the target surface in the flat configuration, facilitating automatic deployment by maximal expansion without the need of any guide surface (right).

we first consider the uniqueness of the deployed configuration, before detailing how to program the desired maximal expansion factor into our discrete triangular linkage.

\subsection{Uniqueness}

The spatially varying maximal extension factor uniquely determines the fully expanded linkage's metric. In other words, the deployed shape is completely determined up to isometric deformation. Does this mean that the metamaterial uniquely encodes the target shape? In general, the answer is no. For instance, the material alone cannot distinguish between "bumps" with negative or positive mean curvature since both produce the same metric distortion. However, in this case our specific deployment methods provide additional regularization: they always produce surfaces of positive mean curvature, eliminating this ambiguity.

Convex surfaces are known to be unique up to global rigid transformations. Surprisingly, the question of whether smooth closed surfaces can be flexible in $\mathbb{R}^{3}$ (i.e., admit infinitesimal deformations preserving the metric) remains an open problem in differential geometry [Ghomi 2017]. So far, no examples have been found, and in practice, all of our examples deployed to their proper target configurations. 

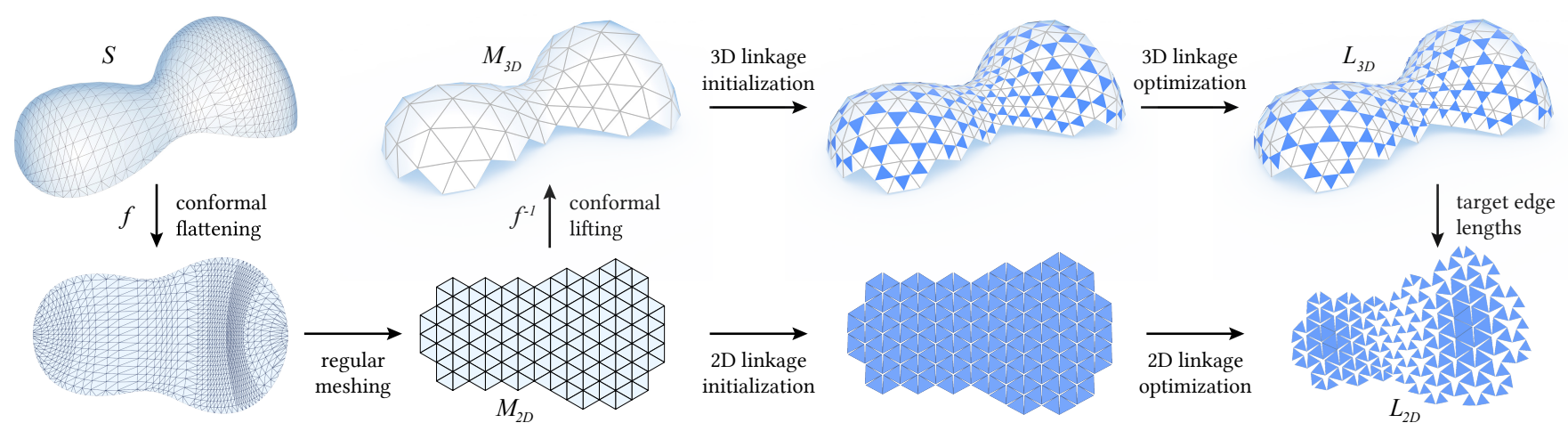

Fig. 8. Sketch of the optimization algorithm for computing the spatially graded auxetic linkage for a given input surface $S$.

\subsection{Auxetic Linkages with Locally-Controlled Stretching}

We now consider how to adapt the regular triangle auxetic linkage structure to impose a spatially-varying upper scaling bound tailored to the conformal scale factor $\lambda_{\text {tgt. }}$. We begin with the following observation: taking the standard linkage pattern (with length stretch factor $\lambda$ in the range $1 \leq \lambda \leq 2$ ) and pre-stretching by $2 / \lambda_{\text {tgt }}$ yields a new material with the stretching bounds $\lambda_{\text {tgt }} / 2 \leq \lambda \leq \lambda_{\text {tgt }}$. Effectively, this pre-stretching limits the amount of additional expansion possible until the fully opened configuration is reached (see Figure 6). This reduces our problem to producing a linkage with a spatially-varying pre-stretch in its flat configuration. The challenge now is to piece together patches with different pre-stretch. As illustrated in Figure 6, this can only be done by scaling the triangles, as will be detailed below.

Figure 7 shows an example and provides a comparison between our spatially graded auxetic linkage and the homogeneous pattern proposed in [Konaković et al. 2016]. Note that the nonuniform linkage structure no longer fully opens or closes in the plane like the regular auxetic linkage could; once any region (hexagonal opening) in the pattern fully opens or fully closes, further expansion/contraction requires spatially varying the stretch factors, inducing curvature that forces the structure into 3D.

\section{MATERIAL OPTIMIZATION}

In this section, we describe our computational workflow and the optimization algorithm for computing the deployable auxetic linkage for a given design surface.

Preprocessing. Our first step is to analyze the input surface to ensure that it satisfies the positive mean curvature requirement. As discussed in Section 3.4, we correct infeasible surfaces by applying mean curvature flow adapted to operate only on regions of non-positive mean curvature(see also Figure 9). We use implicit integration for the flow as proposed by [Desbrun et al. 1999]:

$$
\left(M^{t}+h L^{t}\right) \mathbf{x}^{t+1}=M^{t} \mathbf{x}^{t}
$$

where $M$ is the mass matrix, $h$ is the step size, $L$ is the positive semidefinite cotan Laplace matrix, $\mathbf{x}$ is a matrix of vertex positions (one row per input surface vertex), and the superscripts indicate the iteration number. We run this flow until convergence updating only the positions of vertices with non-positive mean curvature.

Given the corrected input surface $S$, our goal now is to find the 2D layout of the triangular linkage that, when deployed to maximal expansion, approximates $S$ as closely as possible. Figure 8 illustrates the main steps of our algorithm.

Conformal Flattening and Remeshing. We first compute a conformal map $f: S \rightarrow \Omega$ from the target surface $S$ to a planar domain $\Omega \subset \mathbb{R}^{2}$ using the methods of [Sawhney and Crane 2017]. We check if the conformal scale factors are within the bounds prescribed by the linkage mechanism, and, if necessary, introduce cone singularities at user-selected locations to reduce scale distortion as described below. Next, we sample the parametric domain $\Omega$ with a regular equilateral triangle mesh $M_{2 \mathrm{D}}$ that defines the base structure of our linkage. The user selects the resolution and orientation of this mesh to match her design intent. Lifting $M_{2 \mathrm{D}}$ onto $S$ by the inverse map $f^{-1}$ yields $M_{3 \mathrm{D}}$.

$3 D$ Linkage Optimization. We now obtain an initial guess for the fully-opened linkage structure by constructing the medial triangle for each triangle in $M_{3 \mathrm{D}}$ (i.e., inscribing a triangle by connecting edge midpoints; see Figure 8). While this initialization is already close to the desired target configuration, the discrete nature of the lifting function introduces inaccuracies that necessitate further optimization. In particular, we need to ensure that the linkage triangles remain equilateral and are maximally expanded everywhere while staying close to the target surface. Fortunately, these objectives can be formulated easily in the context of the projective approach of [Bouaziz et al. 2012]. Specifically, to obtain the linkage's curved target configuration $L_{3 \mathrm{D}}$ we minimize an energy function $E_{\mathrm{L} 3 \mathrm{D}}$ defined as the sum of three different objective terms over the vertex positions $\mathbf{x}$,

$$
E_{\mathrm{L} 3 \mathrm{D}}(\mathbf{x})=\omega_{1} E_{\text {expand }}(\mathbf{x})+\omega_{2} E_{\text {equi }}(\mathbf{x})+\omega_{3} E_{\text {design }}(\mathbf{x}),
$$

with weights $\omega_{i}$. Each term can be formulated as a sum of constraint proximity functions of the form $\phi\left(\mathbf{x}_{c}\right)=\left\|\mathbf{x}_{c}-P\left(\mathbf{x}_{c}\right)\right\|_{2}^{2}$, where $\mathbf{x}_{c}$ is the vertex set involved in the specific constraint, and $P$ denotes the projection operator to the constraint set, as detailed below. 
We observe that in the fully expanded state, the hexagonal openings formed by the linkage must attain maximum area. By Cramer's theorem [Niven 1981, p. 236], this maximum is achieved when all vertices of the opening lie on a circle.

We thus introduce the expansion term

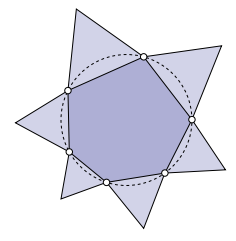

$$
E_{\text {expand }}=\sum_{h \in H}\left\|\mathbf{x}_{h}-P_{C}\left(\mathbf{x}_{h}\right)\right\|_{2}^{2},
$$

where $h$ is an index set of vertices in a particular hexagonal opening, and $H$ is the collection of all such index sets in the linkage. $P_{C}\left(\mathbf{x}_{h}\right)$ defines the projection to the circle closest to the vertices of $\mathbf{x}_{h}$ computed as described in [Bouaziz et al. 2012].

Contrary to the uniform pattern used in [Konaković et al. 2016], our linkage triangles need to vary in scale to introduce spatially varying maximal expansion. In order to let triangles scale freely but keep their equilateral shape, we introduce the energy

$$
E_{\text {equi }}=\sum_{t \in T}\left\|\mathbf{x}_{t}-P_{T}\left(\mathbf{x}_{\mathbf{t}}\right)\right\|_{2}^{2},
$$

where $t$ is the index set of the vertices of a triangle, $T$ is the set of all linkage triangles, and $P_{T}$ is the projection to the closest equilateral triangle, computed using shape matching as described in [Umeyama 1991].

Finally, to keep the linkage close to the design surface, we apply positional constraints of the form

$$
E_{\text {design }}=\sum_{v \in V}\left\|\mathbf{x}_{v}-P_{S}\left(\mathbf{x}_{v}\right)\right\|_{2}^{2},
$$

where $v$ is a vertex index, $V$ is the set of all linkage vertices, and $P_{S}$ defines the projection to the closest point on $S$.

The minimization of $E_{\mathrm{L} 3 \mathrm{D}}$ then follows the typical local/global iteration strategy (see also [Sorkine and Alexa 2007]): the local step computes all the constraint projections involved in the objective terms for the fixed current vertex positions; the global step subsequently solves for the optimal vertex positions keeping the constraint projections fixed. Details on the precise definitions of the projection operators and the corresponding numerical solver implementations can be found in [Bouaziz et al. 2012] and [Deuss et al. 2015].

$2 D$ Linkage Optimization. The 3D optimization provides us with the curved target configuration $L_{3 \mathrm{D}}$ of the linkage in its fully opened state. Now we need to find the contracted linkage in the plane that defines the material rest state to be fabricated. We formulate this problem as a second projective optimization. We first apply the necessary topological cuts to convert $M_{2 \mathrm{D}}$ into a regular triangular linkage $L_{2 \mathrm{D}}$ with uniform triangle sizes (Figure 8). Note that this flat linkage has a one-to-one vertex correspondence with the deployed linkage $L_{3 \mathrm{D}}$. Next, we optimize the $2 \mathrm{D}$ vertex coordinates $\mathbf{u}$ of $L_{2 \mathrm{D}}$ so that the triangles assume the edge lengths of $L_{3 \mathrm{D}}$. This is again easily implemented using a projective edge length constraint of the form

$$
E_{\text {edge }}=\sum_{(i, j) \in E}\left\|\left(\mathbf{u}_{i}-\mathbf{u}_{j}\right)-P_{E}\left(\mathbf{u}_{i}, \mathbf{u}_{j}\right)\right\|_{2}^{2},
$$

where $(i, j)$ denotes the vertex indices of an edge and $E$ is the set of edges of the linkage. The operator

$$
P_{E}\left(\mathbf{u}_{i}, \mathbf{u}_{j}\right)=\frac{\left\|\mathbf{x}_{i}-\mathbf{x}_{j}\right\|}{\left\|\mathbf{u}_{i}-\mathbf{u}_{j}\right\|}\left(\mathbf{u}_{i}-\mathbf{u}_{j}\right)
$$

projects to the closest edge with target length $\left\|\mathbf{x}_{i}-\mathbf{x}_{j}\right\|$ of the corresponding edge in the 3D linkage $L_{3 \mathrm{D}}$. We also add the nonpenetration constraint proposed in [Konaković et al. 2016] to avoid collisions in the $2 \mathrm{D}$ state. The final optimized linkage $L_{2 \mathrm{D}}$ then defines the flat auxetic surface material that deploys to the desired target state.

Algorithm Parameters. Our implementation of the projective constraint solver is based on the open-source library provided by [Deuss et al. 2015]. We set the weights in (2) to $\omega_{1}=\omega_{2}=100$ and $\omega_{3}=1$ and apply between 100 to 600 iterations, depending on the mesh resolution. Total computation time for $3 \mathrm{D}$ and $2 \mathrm{D}$ optimization for a linkage with $8 \mathrm{k}$ triangles is 1.8 minutes on a standard desktop computer with $4.2 \mathrm{GHz}$ computed on a single core.

Cone Singularities. When the conformal scale factors exceed the maximal expansion limits of the auxetic linkage, we need to insert cone singularities in the conformal map to reduce scale distortion. Singularities can also be mandated by the input surface's topology (to satisfy the Gauss-Bonnet theorem). These singularities correspond to boundary vertices of $M_{2 \mathrm{D}}$ where the incident boundary curves (seams) close up when lifted to $M_{3 \mathrm{D}}$ by the conformal map.

Because conformal maps preserve angles, for the surface to close up and form a regular equilateral triangle mesh when lifted to $M_{3 \mathrm{D}}$, the sum of triangle angles around the singular vertex in $M_{2 \mathrm{D}}$ referred to as the cone angle-must be an integer multiple of $\frac{\pi}{3}$. In the inset figure, we show an example with cone angle $\frac{5 \pi}{3}$

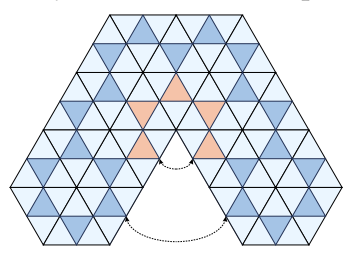
and see how the equilateral triangle mesh (and an inscribed linkage) will properly stitch together when lifted to $M_{3 D}$. Figure 10 shows examples with singularities of cone angle $\frac{4 \pi}{3}$ and $\frac{5 \pi}{3}$.

In-plane Opening. In case the computed scale factors do not fully cover the maximal admissible range, the resulting $2 \mathrm{D}$ linkage can still be expanded in the plane until one hexagonal opening is fully opened-or contracted until one opening is fully closed-as shown in the inset.

We leverage this property for the fabrication result in Figure 1 to reduce the material stresses at the triangle joints during inflation by preopening the linkage as much as possible; this minimizes the rotation necessary to achieve the fully expanded configuration. In the optimization, we add an additional angle

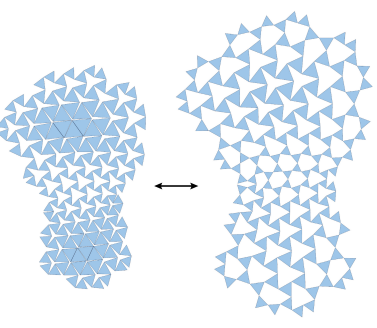
constraint [Deng et al. 2015] with a low weight that either tries to expand or contract the linkage in the flat configuration, depending on the user's preference. 

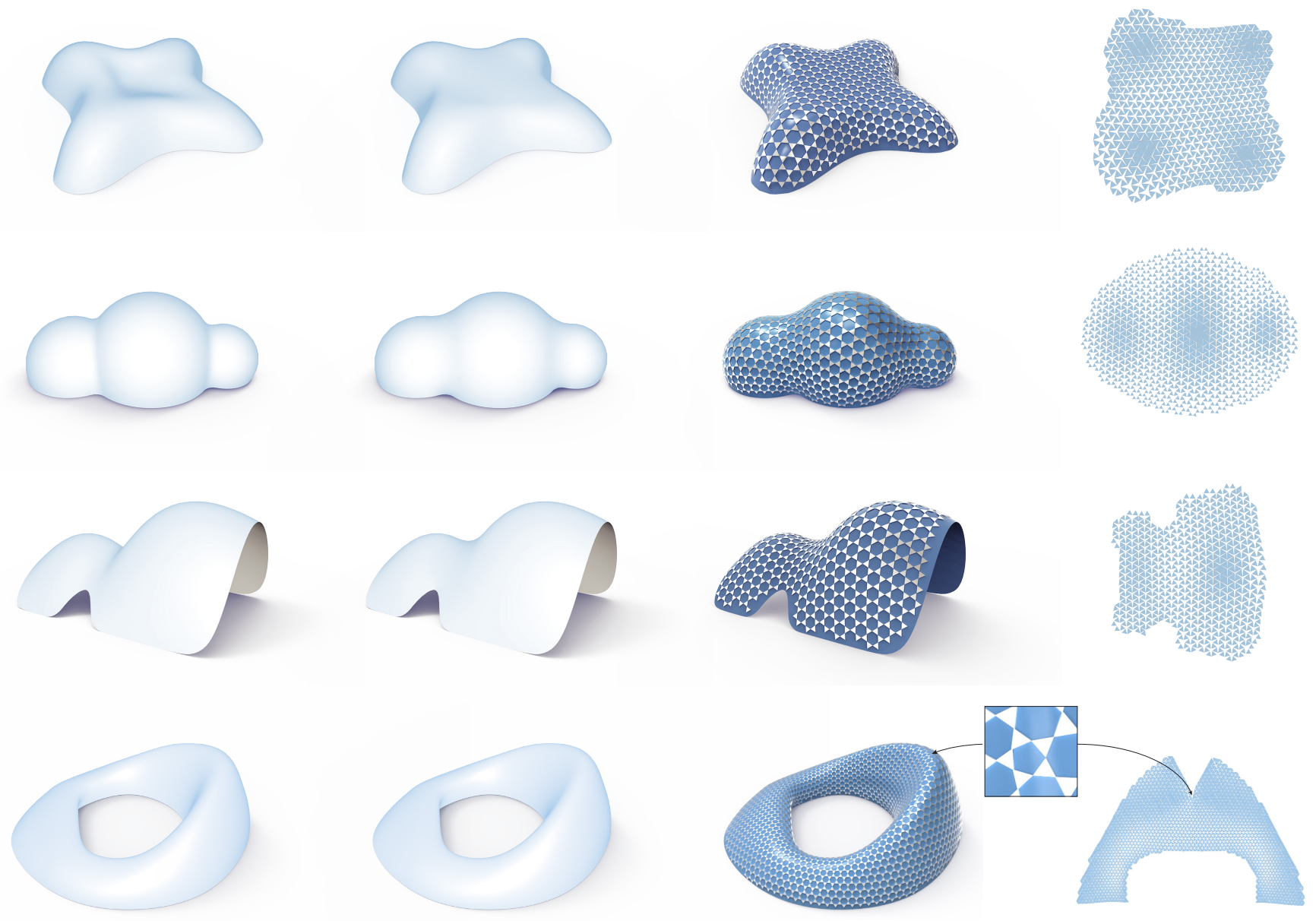

Fig. 9. From left to right: input design surface, modified surface with positive mean curvature everywhere, optimized linkage in deployed state, 2D rest state of flat-fabricatable material. In the bottom row, a singularity of cone angle $\frac{5 \pi}{3}$ is introduced to bring the conformal scale factors to lie within the admissible range.

Filling in the Surface. If the user desires a deployed surface without holes, the hexagonal openings in the fully expanded linkage can be filled in by layering four sheets offset from each other:
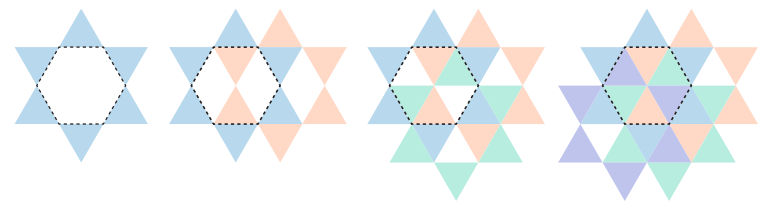

However, simply creating copies of the optimized linkage $L_{3 \mathrm{D}}$ and shifting them does not work: this would effectively translate the deployed surface itself and also would lead to triangles imperfectly fitting the hexagonal holes due to the varying scale factors. Instead, these sheets must be designed by offsetting copies of $M_{2 \mathrm{D}}$ in the parametric domain and lifting/optimizing them in 3D. Figure 4 shows an example of a surface filled in with this method.

Verification by Simulation. Recall that the above optimization maximizes surface expansion of the linkage on a target surface of positive mean curvature. In the smooth setting, our analysis in Section 3 shows that this defines a deployable target surface under inflation or gravity. To verify that this observation also holds in the discrete case, and that the computed linkage does indeed define a steady state under inflation or gravitational loading, we apply a physics-based simulation. We use the same projective approach as we did for linkage design, using only edge length constraints to keep triangles rigid and positional constraints to fix the boundary. We augment this optimization with dynamics as proposed in [Bouaziz et al. 2014] by applying forces on the linkage vertices. For inflation, the force vectors are oriented along the surface normal, for gravity along the fixed negative vertical axis.

Our experiments confirm that the linkages properly deploy, reaching an equilibrium configuration very close to $L_{3 \mathrm{D}}$. For each of our examples, we compute the maximal distance between vertices in $L_{3 \mathrm{D}}$ and their corresponding pairs in the equilibrium linkage. We then compute a maximum relative vertex deviation for each model by dividing this distance by the length of the bounding box diagonal. 

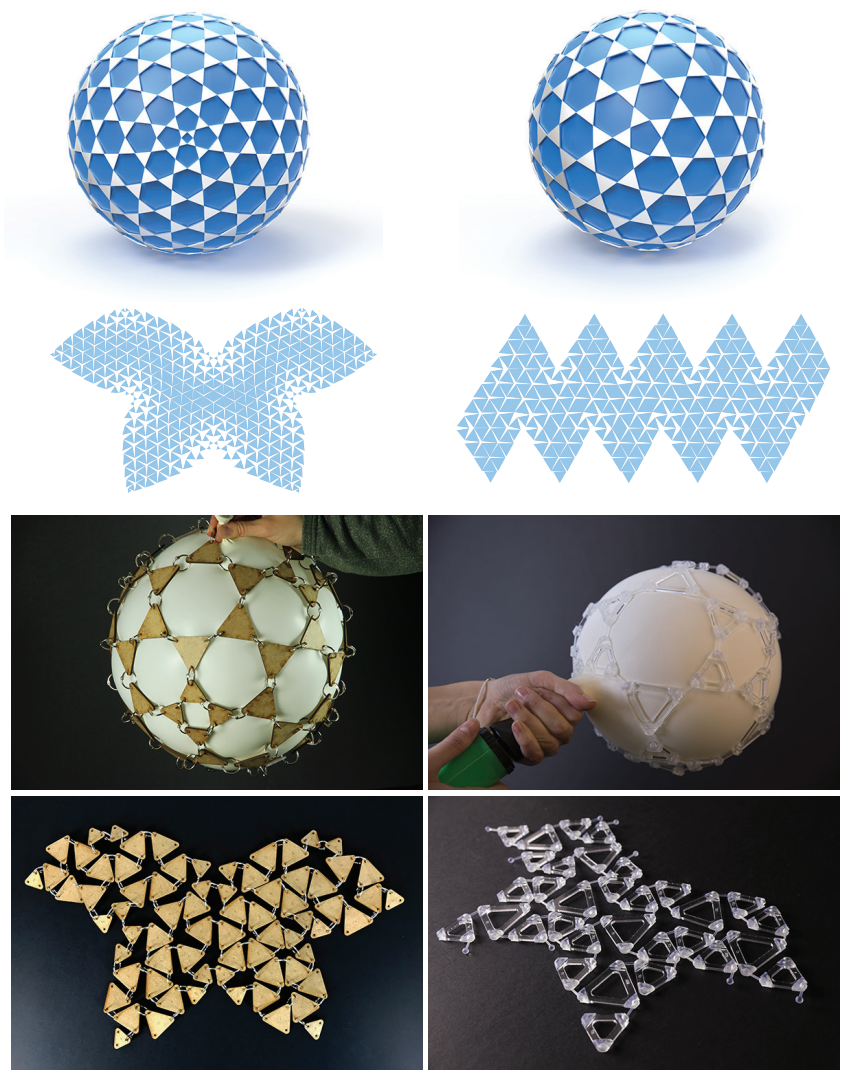

Fig. 10. Cone singularities are required when modeling closed surfaces such as the sphere. The simulated models at the top depict examples with cone angles of $4 \pi / 3$ and $5 \pi / 3$, respectively. At the bottom, lower-resolution fabricated prototypes with cone angles of $4 \pi / 3$ are shown, one fabricated by laser cutting with triangles connected by rings, one 3D printed with ball joint connections. The surfaces have been closed manually along the boundary elements prior to inflation.

The model with the worst relative deviation has a maximal vertex distance of 0.0513 and a bounding box diagonal of 28.5, giving a relative deviation of 0.0018 . In all cases, the differences are nearly imperceptible.

\section{RESULTS}

We verify our material design and optimization approach with a number of numerical and physical experiments, illustrating a range of potential application fields with different materials and usage domains. Figure 9 shows several examples of our deployable auxetic surfaces computed with the optimization algorithm described in Section 5 .

Fabricated Prototypes. Figure 1 shows how one can deploy a doubly-curved freeform surface from a single flat sheet of material. The expansive forces for deployment are created by a generic rubber balloon that is inflated against the support plane. As the balloon is pumped with air, it presses against and deforms the linkage until the target shape is reached at maximal stretch. The balloon has no information about the target shape, which is solely encoded in the linkage pattern computed by our algorithm. Note that while the inflated surface has positive mean curvature everywhere as required by our analysis, both positive and negative Gaussian curvature are present in the target shape. Figure 10 shows how cone singularities are introduced when inflating closed surfaces. The structure of Figure 11 illustrates how gravitational forces can be used to deploy the target shape from the flat rest configuration. The resulting structure is in tension everywhere and can thus be used for physical form-finding of self-supporting structures in analogy to the famous approach of Antoni Gaudi [Fernandez 2006].

Application Case Studies. Figure 2 illustrates an application in personalized medicine, where a deployable freeform coronary stent can be customized to a specific patient. The stent is fabricated as a flat structure, then rolled into a thin cylinder. When inflated, the stent adopts the desired freeform shape to best advance blood flow in the critical artery. Figure 3 highlights an application in deployable architecture to construct a relocatable, semi-permanent structure. Compared to the simple geometries of existing inflatable structures, our approach supports a broader class of freeform shapes, which allows adapting the structure to the design-specific interior space requirements. Figure 4 illustrates how multiple layers of our programmable auxetic material combine to create the approximately closed surfaces of a freeform chair (deployed by gravity).

\section{LIMITATIONS AND FUTURE WORK}

Our current deployment strategies using inflation or gravity can only actuate a subset of the surfaces realizable with a graded auxetic linkage: those with positive mean curvature. Adding additional constraints-for example, in the form of strings connecting certain vertices and thus preventing expansion towards positive mean curvature-can enlarge the space of deployable shapes. It is an interesting question for future work to find a minimal set of such constraints for a given target surface.

For closed surfaces and surfaces requiring singularities, we must introduce cuts to flatten the material to the plane (see Figure 10 and bottom row of Figure 9). While this retains the benefits of planar fabrication, the deployment becomes more complex, as the material has to be re-connected along the cut seam prior to actuation.

Our results confirm that we obtain a close approximation of the target shape even for relatively coarse resolutions of the linkage. However, although we have observed several connections to conformal mapping theory that inform our optimization algorithms, we currently do not have a discrete theory for the geometry of graded auxetic linkages. Developing such a theory in the context of discrete differential geometry is an exciting avenue for future work.

While our fabricated prototypes provide a proof-of-concept for the physical realizability of our designs, we do not address important fabrication-related issues at different scales. In particular, it is crucial for robust deployment to optimize the joints connecting the linkage triangles. It also would be interesting to test techniques for permanently rigidifying the deployed structure. We hope that our work can stimulate new research in material science, mechanical engineering, and architectural construction to study these questions in more detail. 

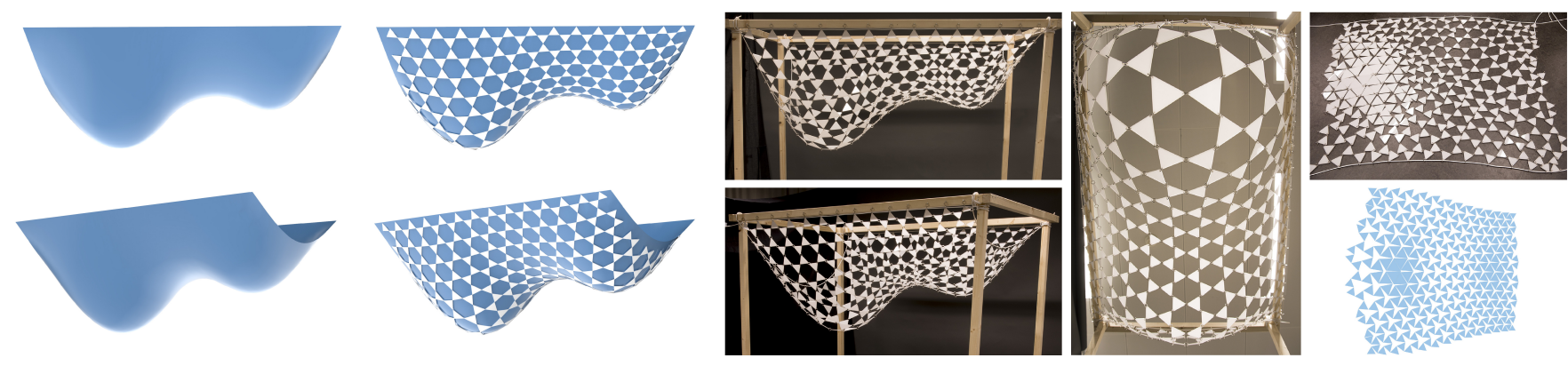

Fig. 11. Deployment via gravity. The auxetic linkage shown on the bottom right has been optimized to match the input design surface on the left. The structure has been assembled in the flat state from individually laser-cut triangles that are connected by metallic rings to enable the rotational motion of the linkage triangles. When lifted onto the rectangular support, the surface automatically deploys into its target shape. Note that boundary vertices are fixed along the long edges of the support rectangle, and connected with strings on the short edges.

\section{CONCLUSION}

Numerous physical objects, such as ship or airplane hulls, building facades, clothing, and many consumer products are fabricated by shaping thin, initially planar materials. The shaping process typically involves bending, stretching, or otherwise deforming the material using a mold or scaffold to guide the deformation towards the desired 3D shape. Deployable structures provide an alternative where the shaping process and resulting target geometry are implicitly encoded in the structure itself. We have shown that spatially graded auxetics are well suited to implement deployable surface structures. Instead of rationalizing a 3D design surface for a given homogeneous material, we spatially optimize the material itself. By carefully controlling the expansion behavior of the material, we directly program the target surface geometry into the flat $2 \mathrm{D}$ rest state. Inflation or gravitational loading then automatically deploys the rest state towards the target, which is assumed when the material cannot expand any further. As a consequence, we can leverage the efficiency of 2D digital fabrication technologies without requiring any additional 3D guide surface. Our deployment strategy is robust and reversible, which supports efficient storage and transport and enables new applications for semi-permanent structures.

The combination of limited-expansion auxetic material with a deployment via inflation or gravity imposes limits on the space of realizable shapes. Our analysis clearly delimits this space and directly informs our computational solution, providing designers with an effective tool to realize new deployable structures not possible before.

\section{ACKNOWLEDGMENTS}

We are grateful to Pavle Konaković for his help with design, fabrication, and renderings. We thank the CMU Geometry Collective members and Gaspard Zoss for insightful discussions. We also thank Liane Makatura, Peng Song, and the anonymous reviewers for their valuable feedback. This work was supported by the NCCR Digital Fabrication, funded by the Swiss National Science Foundation, NCCR Digital Fabrication Agreement \#51NF40-141853, NSF Award 1717320, and gifts from Autodesk Research and Adobe Research.

\section{REFERENCES}

Bernd Bickel, Moritz Bächer, Miguel A. Otaduy, Hyunho Richard Lee, Hanspeter Pfister, Markus Gross, and Wojciech Matusik. 2010. Design and Fabrication of Materials with Desired Deformation Behavior. ACM Trans. Graph. 29, 4, Article 63 (July 2010), 10 pages.

Sofien Bouaziz, Mario Deuss, Yuliy Schwartzburg, Thibaut Weise, and Mark Pauly. 2012. Shape-Up: Shaping Discrete Geometry with Projections. Comput. Graph. Forum 31, 5 (2012), 1657-1667.

Sofien Bouaziz, Sebastian Martin, Tiantian Liu, Ladislav Kavan, and Mark Pauly. 2014. Projective Dynamics: Fusing Constraint Projections for Fast Simulation. ACM Trans. Graph. 33, 4, Article 154 (July 2014), 11 pages.

Isaac Chao, Ulrich Pinkall, Patrick Sanan, and Peter Schröder. 2010. A Simple Geometric Model for Elastic Deformations. ACM Trans. Graph. 29, 4, Article 38 (July 2010), 6 pages.

Xiang Chen, Changxi Zheng, Weiwei Xu, and Kun Zhou. 2014. An Asymptotic Numerical Method for Inverse Elastic Shape Design. ACM Trans. Graph. 33, 4, Article 95 (July 2014), 11 pages.

Fionnuala Connolly, Conor J. Walsh, and Katia Bertoldi. 2017. Automatic design of fiber-reinforced soft actuators for trajectory matching. Proceedings of the National Academy of Sciences 114, 1 (2017), 51-56.

Bailin Deng, Sofien Bouaziz, Mario Deuss, Alexandre Kaspar, Yuliy Schwartzburg, and Mark Pauly. 2015. Interactive Design Exploration for Constrained Meshes. Computer-Aided Design 61 (2015), 13-23.

Mathieu Desbrun, Mark Meyer, Peter Schröder, and Alan H. Barr. 1999. Implicit Fairing of Irregular Meshes Using Diffusion and Curvature Flow. In Proceedings of the 26th Annual Conference on Computer Graphics and Interactive Techniques (SIGGRAPH '99). ACM Press/Addison-Wesley Publishing Co., New York, NY, USA, 317-324.

Mario Deuss, Anders Holden Deleuran, Sofien Bouaziz, Bailin Deng, Daniel Piker, and Mark Pauly. 2015. ShapeOp-A Robust and Extensible Geometric Modelling Paradigm. Springer International Publishing, 505-515.

Günay Doğan and Ricardo H. Nochetto. 2012. ESAIM: Mathematical Modelling and Numerical Analysis 46, 1 (2012), 59-79.

Levi H. Dudte, Etienne Vouga, Tomohiro Tachi, and L. Mahadevan. 2016. Programming curvature using origami tessellations. Nature Materials 15, 5 (2016), 583-588.

J. Eells and J. C. Wood. 1976. Restrictions on Harmonic Maps of Surfaces. Topology 15 (1976), 263-266.

Santiago Fernandez. 2006. Structural Design in the Work of Gaudi. 49 (12 2006), 324-339.

Jan Friedrich, Sven Pfeiffer, and Christoph Gengnagel. 2018. Locally Varied Auxetic Structures for Doubly-Curved Shapes. Springer Singapore, Singapore, 323-336.

C.J. Gantes. 2001. Deployable Structures: Analysis and Design. WIT Press.

Mohammad Ghomi. 2017. Open Problems in Geometry of Curves and Surfaces. http: //people.math.gatech.edu/ ghomi/Papers/op.pdf. (Oct. 2017). Accessed: 2017-12-12.

Ruslan Guseinov, Eder Miguel, and Bernd Bickel. 2017. CurveUps: Shaping Objects from Flat Plates with Tension-actuated Curvature. ACM Trans. Graph. 36, 4, Article 64 (July 2017), 12 pages.

Caigui Jiang, Chengcheng Tang, Amir Vaxman, Peter Wonka, and Helmut Pottmann. 2015. Polyhedral Patterns. ACM Trans. Graph. 34, 6, Article 172 (Oct. 2015), 12 pages.

Sun-Jeong Kim, Soo-Kyun Kim, and Chang-Hun Kim. 2002. Discrete differential error metric for surface simplification. In 10th Pacific Conference on Computer Graphics and Applications, 2002. Proceedings. 276-283.

Mina Konaković, Keenan Crane, Bailin Deng, Sofien Bouaziz, Daniel Piker, and Mark Pauly. 2016. Beyond Developable: Computational Design and Fabrication with 
Auxetic Materials. ACM Trans. Graph. 35, 4, Article 89 (July 2016), 11 pages.

W. Y. Lam. 2017. Minimal surfaces from infinitesimal deformations of circle packings ArXiv e-prints (Dec. 2017).

Ying Liu, Brandi Shaw, Michael D. Dickey, and Jan Genzer. 2017. Sequential self-folding of polymer sheets. Science Advances 3, 3 (2017).

Li-Ke Ma, Yizhong Zhang, Yang Liu, Kun Zhou, and Xin Tong. 2017. Computational Design and Fabrication of Soft Pneumatic Objects with Desired Deformations. ACM Trans. Graph. 36, 6, Article 239 (Nov. 2017), 12 pages.

I. Niven. 1981. Maxima and Minima Without Calculus. Number v. 6 in Dolciani Mathematical Expositions. Mathematical Association of America.

Jifei Ou, Mélina Skouras, Nikolaos Vlavianos, Felix Heibeck, Chin-Yi Cheng, Jannik Peters, and Hiroshi Ishii. 2016. aeroMorph - Heat-sealing Inflatable Shape-change Materials for Interaction Design. In Proceedings of the 29th Annual Symposium on User Interface Software and Technology (UIST '16). ACM, New York, NY, USA 121-132.

Johannes T. B. Overvelde, Twan A. de Jong, Yanina Shevchenko, Sergio A. Becerra, George M. Whitesides, James C. Weaver, Chuck Hoberman, and Katia Bertoldi. 2016 A three-dimensional actuated origami-inspired transformable metamaterial with multiple degrees of freedom. 7 (11 03 2016).

Julian Panetta, Qingnan Zhou, Luigi Malomo, Nico Pietroni, Paolo Cignoni, and Denis Zorin. 2015. Elastic Textures for Additive Fabrication. ACM Trans. Graph. 34, 4 Article 135 (July 2015), 12 pages.

Jesús Pérez, Miguel A. Otaduy, and Bernhard Thomaszewski. 2017. Computational Design and Automated Fabrication of Kirchhoff-plateau Surfaces. ACM Trans. Graph 36, 4, Article 62 (July 2017), 12 pages.

Jesús Pérez, Bernhard Thomaszewski, Stelian Coros, Bernd Bickel, José A. Canabal, Robert Sumner, and Miguel A. Otaduy. 2015. Design and Fabrication of Flexible Rod Meshes. ACM Trans. Graph. 34, 4, Article 138 (July 2015), 12 pages.

Ahmad Rafsanjani and Damiano Pasini. 2016. Bistable auxetic mechanical metamaterials inspired by ancient geometric motifs. Extreme Mechanics Letters 9 (2016), 291 - 296.

Dan Raviv, Wei Zhao, Carrie McKnelly, Athina Papadopoulou, Achuta Kadambi, Boxin Shi, Shai Hirsch, Daniel Dikovsky, Michael Zyracki, Carlos Olguin, Ramesh Raskar, and Skylar Tibbits. 2014. Active Printed Materials for Complex Self-Evolving Deformations. 4 (18 12 2014).

Rohan Sawhney and Keenan Crane. 2017. Boundary First Flattening. ACM Trans. Graph. 37, 1, Article 5 (Dec. 2017), 14 pages.

Krishna Kumar Saxena, Raj Das, and Emilio P. Calius. 2016. Three Decades of Auxetics Research - Materials with Negative Poisson's Ratio: A Review. Advanced Engineering Materials 18, 11 (2016), 1847-1870.

Alexander Schiftner, Mathias Höbinger, Johannes Wallner, and Helmut Pottmann. 2009. Packing Circles and Spheres on Surfaces. ACM Trans. Graph. 28, 5, Article 139 (Dec 2009), 8 pages.

Christian Schumacher, Bernd Bickel, Jan Rys, Steve Marschner, Chiara Daraio, and Markus Gross. 2015. Microstructures to Control Elasticity in 3D Printing. ACM Trans. Graph. 34, 4, Article 136 (July 2015), 13 pages.

Mélina Skouras, Bernhard Thomaszewski, Bernd Bickel, and Markus Gross. 2012. Computational Design of Rubber Balloons. Comput. Graph. Forum 31, 2pt4 (May 2012), 835-844.

Mélina Skouras, Bernhard Thomaszewski, Peter Kaufmann, Akash Garg, Bernd Bickel, Eitan Grinspun, and Markus Gross. 2014. Designing Inflatable Structures. ACM Trans. Graph. 33, 4, Article 63 (July 2014), 10 pages.

Olga Sorkine and Marc Alexa. 2007. As-rigid-as-possible Surface Modeling. In Proceedings of the Fifth Eurographics Symposium on Geometry Processing (SGP '07) Eurographics Association, 109-116.

Hideshi Tomita, Takashi Higaki, Toshiki Kobayashi, Takanari Fujii, and Kazuto Fujimoto. 2016. Stenting for curved lesions using a novel curved balloon: Preliminary experimental study. 66 (8 2016), 120-124. Issue 2 .

Shinji Umeyama. 1991. Least-Squares Estimation of Transformation Parameters Between Two Point Patterns. IEEE Trans. Pattern Anal. Mach. Intell. 13, 4 (1991), 376-380.

Amir Vaxman, Christian Müller, and Ofir Weber. 2017. Regular Meshes from Polygonal Patterns. ACM Trans. Graph. 36, 4, Article 113 (July 2017), 15 pages.

Changxi Zheng, Timothy Sun, and Xiang Chen. 2016. Deployable 3D Linkages with Collision Avoidance. In Proceedings of the ACM SIGGRAPH/Eurographics Symposium on Computer Animation (SCA '16). Eurographics Association, Aire-la-Ville, Switzerland, Switzerland, 179-188.

Bo Zhu, Mélina Skouras, Desai Chen, and Wojciech Matusik. 2017. Two-Scale Topology Optimization with Microstructures. ACM Trans. Graph. 36, 5, Article 164 (July 2017), 16 pages. 